\title{
Schwannoma melanótico: Reporte de un caso y revisión de la literatura
}

\author{
Drs. María Ignacia Labbé $A^{(1)}$, Gonzalo Miranda $G^{(2)}$, Facundo Las Heras $A^{(3)}$.
}

1. Médico Cirujano

2. Médico Neuroradiólogo. Servicio de Radiología, Hospital Clínico Universidad de Chile - Chile

3. Médico Anátomo-Patólogo, PhD. Servicio de Anatomía Patológica, Hospital Clínico Universidad de Chile - Chile.

\section{Melanotic schwannoma: A case report and literature review}

\begin{abstract}
Schwannomas are benign neoplastic lesions which originate from Schwann cells. A rare variant is the melanotic schwannoma. Accurate discrimination of this entity may be difficult due to differential diagnosis with malignant tumors, especially with metastatic melanoma, which has a potential ominous prognosis and a radically different treatment. We report the case of a 60-year-old woman with neurological sensorimotor involvement, presenting progressive caudo-cranial involvement more pronounced on the right side. MRI showed an intradural extramedullary mass with hyperintense signal intensity on T1 and T2 images, whereas no significant increase in signal intensity of the spinal cord was observed. Tumor was resected and sent for anatomopathological analysis which revealed a non-psammomatous melanotic schwannoma. Keywords: Intradural extramedullar mass, Melanotic schwannoma, MRI.
\end{abstract}

Resumen: Los schwannomas son lesiones neoplásicas benignas derivadas de las células de Schwann. Una variante poco frecuente es el schwannoma melanótico. El diagnóstico de esta entidad reviste complejidad debido al diagnóstico diferencial con tumores malignos, especialmente el melanoma metastásico, teniendo este último un potencial pronóstico ominoso y un tratamiento radicalmente distinto. Se realizó una revisión de literatura en relación a un caso clínico de una mujer de 60 años con compromiso neurológico de tipo sensitivo-motor, progresivo caudo-craneal mayor a derecha. La RM demostró una masa intradural extramedular, con hiperintensidad en secuencias T1 e hiposeñal en T2, sin aumento de señal significativo de la médula espinal. El tumor fue resecado revelando en el estudio anatomopatológico un schwannoma melanótico, no psammomatoso. En relación al caso clínico, pudimos observar la importancia del estudio imaginológico con RM y su confirmación anatomopatológica. Recalcamos además, la necesidad del seguimiento a largo plazo.

Palabras clave: Masa intradural extramedular, RM, Schwannoma melanótico.

Labbé MI y cols. Schwannoma melanótico: Reporte de un caso y revisión de la literatura. Rev Radiol 2012; 18(1): 5-8. Correspondencia: Dra. María Ignacia Labbé A. / ignacialabbe@gmail.com

Trabajo recibido el 01 de noviembre de 2011, aceptado para publicación el 12 de enero de 2012.

\section{Introducción}

Los schwannomas son lesiones neoplásicas benignas derivadas de las células de Schwann, células constituyentes de la vaina nerviosa ${ }^{(1)}$. La variante menos frecuente dentro de este tipo de neoplasias es el schwannoma melanótico ${ }^{(2)}$. Este puede presentarse de forma esporádica o asociado al complejo de Carney ${ }^{(3)}$.

El diagnóstico de esta entidad reviste complejidad debido al diagnóstico diferencial con tumores malignos, especialmente el melanoma metastásico, teniendo este último un pronóstico y tratamiento radicalmente distinto(4). Este último, a diferencia del schwannoma, sigue un curso maligno, requiriendo resección amplia para evitar la recurrencia local y eventual potencial metastásico ${ }^{(5)}$.
Presentamos aquí un caso clínico de schwannoma melanótico, incluyendo una extensa revisión de la literatura sobre este tema.

\section{Caso clínico}

Mujer de 60 años con antecedentes de una parálisis facial derecha antigua y una trombosis de la vena central de la retina del ojo izquierdo. Entre otros antecedentes presenta hipertensión, dislipidemia y obesidad. Sin antecedentes familiares de importancia.

Consulta por un cuadro de aproximadamente un año de evolución de paresia e hipoestesia en extremidades inferiores, mayor a derecha, progresando a extremidades superiores. Un mes previo al ingreso evoluciona con una importante limitación de la marcha 
y torpeza motora en la mano derecha.

Al examen neurológico de ingreso se evidencia una parálisis facial periférica derecha y una paresia motora M2 en las cuatro extremidades, con reflejos osteotendíneos conservados y extensor plantar a izquierda. Se constata también una hipoestesia multimodal en las cuatro extremidades, sin alteración cerebelosa y signos meníngeos negativos.

Debido a la sospecha clínica de una lesión medular alta se realizó una resonancia magnética (RM), en la cual se demostró una lesión expansiva intradural extramedular, que mostraba una señal espontáneamente hiperintensa en secuencias T1 y de marcada hiposeñal en secuencias T2, sin aumento de señal significativa de la médula espinal, la cual aparecía francamente comprimida por esta masa (Figura 1). Dentro de los diagnósticos diferenciales radiológicos se plantearon meningioma, quiste epidermoide/dermoide, quiste aracnoides con contenido hemorrágico y melanoma metastásico leptomeníngeo.

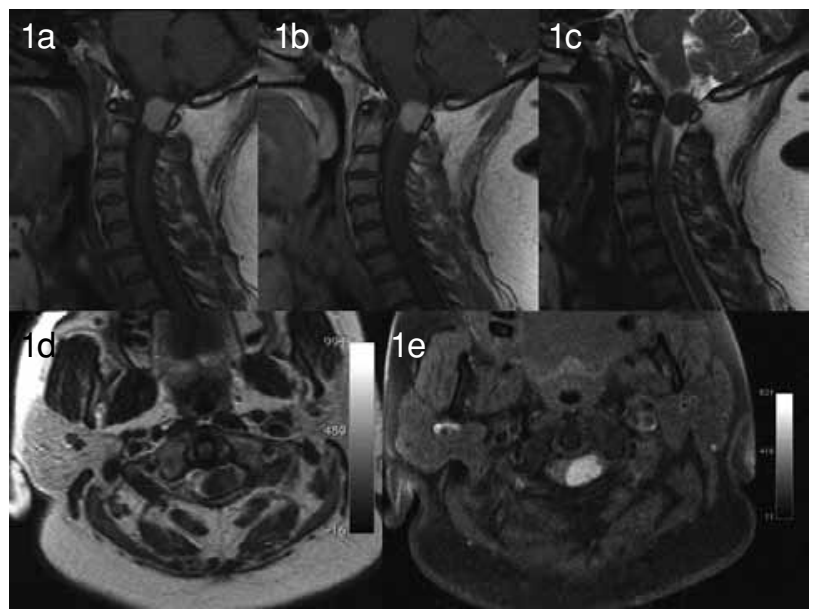

Figura 1. Resonancia magnética de columna cervical. Secuencias sagitales ponderadas en T1 (a), T1 gadolinio (b), T2 Spin eco (c) y axiales T2 Spin eco (d) y T1 FatSat gadolinio (e). Lesión intradural extra medular espontáneamente hiperintensa en secuencias T1 y de marcada baja señal en secuencias T2 que determina una importante compresión de la médula espinal. Dada su señal hiperintensa en secuencias $T 1$ resulta difícil determinar si la masa realza con el uso de gadolinio.

Se realizó una craneotomía suboccipital con resección de arco posterior $\mathrm{C} 1$ y resección del tumor intradural. La paciente evolucionó favorablemente y fue dada de alta.

El tumor resecado fue procesado para estudio anatomopatológico. Secciones histológicas fueron obtenidas del bloque en parafina y consecuentemente teñidas con hematoxilina y eosina. El diagnóstico concluyó un schwannoma melanótico, no psammomatoso (Figura 2).

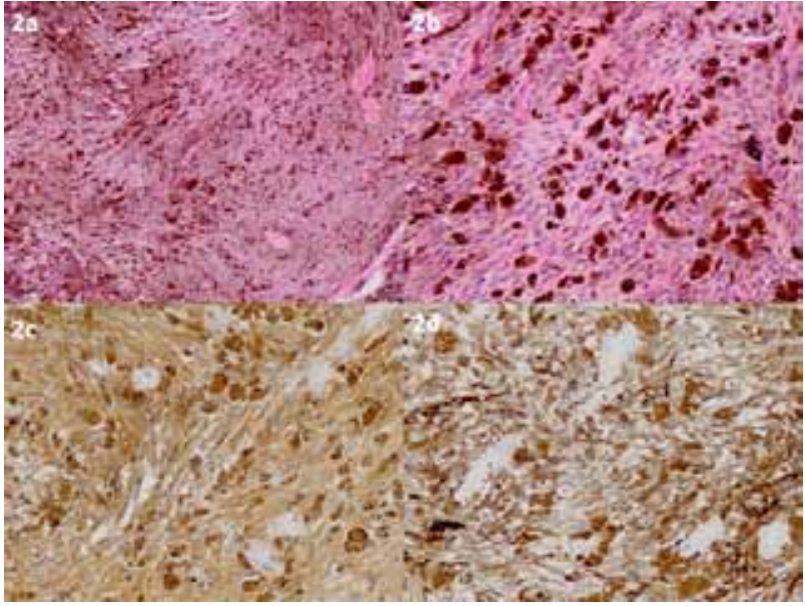

Figura 2. a: Histológicamente se evidencia tumor de celularidad moderada a acentuada, con extensos depósitos de melanina (HE; 100X). b: A mayor aumento, las células son elongadas, sin atipias ni mitosis, asociadas a abundante pigmento melánico (HE; 200X). c:Inmunohistoquímica para proteína S100 muestra positividad acentuada en las células neoplásicas (S100; 200X). d:Vimentina muestra también extensa positividad, revelando la naturaleza mesenquimática de la lesión (Vimentina; 200X).

\section{Análisis y diagnóstico diferencial}

Desde el punto de vista imagenológico, al encontrarnos con una masa intrarraquídea se debe primero definir si la lesión es intra o extramedular, ya que el diagnóstico diferencial es distinto en cada caso. Este caso se presenta como una masa intradural extramedular (Figura 1). Dentro de las principales lesiones extradurales debemos considerar schwannomas, meningiomas, ependimoma del cono, metástasis, quistes aracnoidales, quistes dermoides-epidermoides, linfoma, paragangliomas y sarcoidosis entre otros ${ }^{(6)}$. Como vemos, el diagnóstico diferencial es bastante extenso, por lo que debemos analizar otras características, tales como la ubicación y relación de la lesión dentro del compartimiento intradural, su señal y realce con el medio de contraste paramagnético.

El presente caso muestra una lesión con características de señal inusuales, con alta señal en secuencias T1 y baja señal en secuencias T2, permitiendo limitar el diagnóstico diferencial.

Dentro de las lesiones con estas características de señal se deben mencionar los tumores productores de melanina. La melanina es una sustancia paramagnética que determina un acortamiento de los tiempos T1, por lo cual las lesiones aparecen hiperintensas en estas secuencias. Es por ello que el diagnóstico diferencial incluye los melanocitomas y las metástasis de melanoma maligno( ${ }^{(6)}$. Es importante, además, destacar que las lesiones agresivas suelen acompañarse por edema vasogénico, hallazgo que no fue observado en nuestro caso.

Otra lesión que puede contener melanina son los 
schwannomas melanóticos. Si bien son de presentación excepcional, se deben tener presentes en el diagnóstico diferencial, especialmente en el segmento cervical y torácico.

También se deben considerar los quistes epidermoides "blancos". Los quistes epidermoides generalmente tienen una señal similar al LCR en secuencias T1 y T2, pero en raras ocasiones muestra una señal elevada en secuencias T1, hallazgo que se debe a la concentración elevada de triglicéridos y ácidos grasos insaturados. En estos casos también se observan hipointensos en secuencias $\mathrm{T2}^{(7)}$.

Otra lesión que ocasionalmente puede tener estas características de señal son los quistes aracnoidales. Estos, si bien característicamente siguen la señal del LCR en todas las secuencias, también pueden sufrir fenómenos hemorrágicos que alteran su composición, apareciendo hiperintensos en secuencias T1 por la presencia de metahemoglobina ${ }^{(6)}$.

\section{Discusión}

Los schwannomas son lesiones neoplásicas benignas frecuentes derivadas de las células de Schwann, constituyentes de la vaina nerviosa. La formación de melanina en esta neoplasia se explica debido a que tanto las células de Schwann y los melanocitos provienen de las células de la cresta neural|(5,8). Entre sus variantes histológicas se incluyen el schwannoma clásico, celular, plexiforme, epitelioide y melanótico ${ }^{(9)}$. Estos últimos son una variante poco frecuente y están compuestos por células productoras de melanina con características estructurales de las células de Schwann ${ }^{(10)}$. Presentan dos subtipos: el esporádico y el schwannoma melanótico psammomatoso (PMS). Un 50\% de los PMS están asociados al complejo de Carney ${ }^{(3,11)}$. Este complejo fue descrito en 1985 por Carney, como un síndrome de herencia autosómica dominante que se caracteriza por presentar mixomas (corazón, piel, mama), pigmentación lentiforme mucocutánea e hiperactividad endocrina (debido a disfunción tiroidea o adenoma hipofisario o síndrome de Cushing, secundario a una hiperplasia nodular primaria adrenocortical) ${ }^{(12,13)}$.

Se manifiesta en diversas localizaciones, incluyendo raíces posteriores de los nervios espinales, generalmente a nivel cervical o torácico, así como también el sistema nervioso central y sistema nervioso autónomo. De manera infrecuente pueden afectar tejidos fuera del sistema nervioso como el mediastino, retroperitoneo, páncreas, parótida y tejido subcutáneo, de manera infrecuente ${ }^{(1)}$.

Sin predilección por sexo, su edad media de presentación es de 38.4 años, abarcando edades desde los 10 hasta los 84 años $^{(9)}$.

Su presentación clínica depende de su localización y ritmo de crecimiento, consultando frecuentemente por dolor y síntomas neurológicos. En el caso de lesiones ubicadas en el canal espinal (extramedular), éstas pueden presentar dolor radicular, disestesias y compromiso sensitivo-motor progresivo.

Se requiere de trabajo multidisciplinario recurriendo a la clínica, citología, inmunohistoquímica y radiología para hacer el diagnóstico adecuado.

El diagnóstico preoperatorio se basa en la RM, donde los schwannomas melanóticos se observan hiperintensos en $\mathrm{T} 1$ debido al efecto paramagnético de la melanina, producto de radicales libres estables ${ }^{(14)}$ e hipointenso en T2, diferenciándose de los tumores no melanóticos que se aprecian de forma inversa en las imágenes.

Macroscópicamente se presentan como una masa sólida, con bordes bien delimitados y, por lo general, de color café y/o negro.

Microscópicamente se observan como lesiones bien delimitadas, sin infiltración a tejidos adyacentes, compuesta por una proliferación fusocelular de moderada densidad con células predominantemente elongadas siendo la minoría de aspecto epitelioide (Figura 2A). Los núcleos son ovales con cromatina vesicular y pequeños nucléolos eosinófilos. En el presente caso se observó sólo leve atipia citológica, con escaso número de vasos de paredes hialinizadas en el estroma (Figura 2 B). Mitosis y necrosis tumoral fueron ausentes. Característicamente, estos tumores presentan abundantes gránulos de melanina intracitoplasmática, positiva a la tinción de Fontana-Masson, confirmando así el diagnóstico de schwannoma melanótico. Estos gránulos tiñen negativo para hierro y PAS, lo que los diferencia de gránulos de hemosiderina y lípidos ${ }^{(4,15)}$. No se observaron cuerpos de Verocay, áreas hipocelulares ni formaciones microquísticas, estructuras presentes en los schwannomas clásicos ${ }^{(4)}$.

El diagnóstico histológico-inmunohistoquímico reviste complejidad, ya que el schwannoma melanótico se asemeja mucho en su morfología a los melanomas metastásicos. Sin embargo, el diagnóstico de melanoma está apoyado por la ausencia de las células dendríticas y la presencia de signos de malignidad ${ }^{(1)}$.

Si hay sospecha de malignidad se buscan características microscópicas agresivas como son el pleomorfismo celular, aumento de la actividad mitótica, ausencia de cápsula fibrosa y zonas de necrosis ${ }^{(15)}$.

Inmunohistoquímicamente son reactivos para los marcadores vimentina (Figura 2C), S-100 (Figura 2D) y Leu 7. La inmunorreactividad para HMB-45 es menos frecuente, no siendo de utilidad diagnóstica, ya que este marcador está presente en el melanoma maligno(16,17).

El schwannoma melanótico por lo general tiene un buen pronóstico ${ }^{(18-22)}$, aunque en ciertos casos siguen un curso incierto, llegando a presentar recurrencias locales y metástasis a pesar de la ausencia de características malignas a nivel histológico. Debido a que el seguimiento de muchos de estos tumores es a corto 
plazo, se pueden pasar por alto algunas recurrencias locales y metástasis. Se ha descrito recurrencias y metástasis tardías, con síntomas que se inician en promedio a los 3.6 años e incluso algunos a los 5-6 años posterior al diagnóstico inicial( ${ }^{(5)}$. Una revisión de 57 casos de schwannomas melanóticos hecha por Vallat-Decouvelaere et al. demostró que el $15 \%$ de ellos presentaban recurrencias locales y $26.3 \%$ se complicaban con metástasis, sólo el $53 \%$ de los casos seguidos por más de 5 años se encontraban libres de enfermedad y el $67.5 \%$ de los casos sólo tenían seguimientos de corto plazo ( $<5$ años). Es por ello que la resección tumoral completa y el seguimiento a largo plazo reviste vital importancia.

\section{Bibliografía}

1. Katati MJ, Martín JM, Massare E, Arjona V. Schwannoma melanocítico.A propósito de un caso y revisión de la literatura. Rev Neurol 2000; 31 (5): 427-430.

2. Er U, Kazanci A, Eyriparmak T, Yigitkanli K, Senveli E. Melanotic schwannoma. J Clin Neurosci. 2007; 14(7): 676-678.

3. Kuchelmeister K, Lotz C, Schonmayr R, Schachenmayr W. Woman in her early fifties with a cervical intraspinal and extraspinal mass lesion. Brain Pathol 2004; 14(4): 453-454, 458-459.

4. Zhang H, Yang G., Chen H., Wei B., Ke Q., Guo H. et al. Clinicopathological, inmunohistochemical and ultrastructural study of 13 cases of melanotic schwannoma. Chin Med J 2005; 118(17): 1451-1461.

5. Vallat-Decouvelaere AV, Wassef M, Lot G, Catala M, Moussalam M, Caruel N, Mikol J. Spinal melanotic schwannoma: a tumour with poor prognosis. Histopathology 1999; 35: 558-566.

6. Osborn A, Ross J, Salzman k. Spine Intradural - Extradural. Expertddx. Brain and Spine 2009; II6 2-II6 36.

7. Li F et al. Hyperdense intracraneal epidermoid cyst: a study of 15 cases. Acta Neurochir 2007; 149(1): 31-39.

8. Baroffio A, Dupin E, Le Douarin NM. Common precursors for neural and mesectodermal derivatives in the cephalic neural crest. Development 1991; 112: 301-305.

9. Jiménez F, Márquez B, Merino J, Serrano M, Gutiérrez F, Rozas M. et al. Schwannoma Melanótico. Presen- tación de un caso y revisión de la literatura. Congreso virtual de anatomía patológica, Marzo 2004, España.

10. Er U, Kazanci A, Eyriparmak T, Yigitkanli K, Senveli E. Melanotic schwannoma. J Clin Neurosci. 2007; 14(7): 676-678.

11. Wilkes D, Mcdermott DA, Basson CT. Clinical phenotypes and molecular genetic mechanisms of Carney complex. Lancet Oncol 2005; 6(7): 501-508.

12. Carney JA. Psammomatous melanotic schwannoma $A$ distinctive, heritable tumor with special associations, including cardiac myxoma and the cushing syndrome. Am J Surg Pathol 1990; 14: 206-222.

13. Carney JA, Stratakis CA. Epithelioid blue nevus and psammomatous melanotic schwannoma: the unusual pigmented skin tumors of the Carney complex. Semin Diagn Pathol 1998; 15: 216-224.

14. Gomori JM, Grossman RI, Shields JA. Choroidal melanomas: correlation of NMR spectroscopy and MR imaging. Radiology 1986; 158: 443-445.

15. Jürgen Schmitz K, Unkel C, Grabellus F, Andreas $\mathrm{H}$, Dirsch $\mathrm{O}$, Neumann A. Melanotic schwannoma of the neck mimicking a malignant melanoma. Eur Arch Otorhinolaryngol 2005; 262: 182-185.

16. Azarpira N, Torabineghad S, Sepidbakht S, Rakei M., Hadi Bagheri M. Cytologic findings in pigmented melanotic schwannoma, A case report. Acta Cytologica 2009; 53: 113-115.

17. Bosman C. Boldrini R, Corsi A. Malignant melanotic schwannoma or schwannian melanoma. Tumori 1995; 81: $208-212$.

18. Gregorios JB, Chou SM, Bay J. Melanotic schwannoma of the spinal cord. Neurosurgery 1982; 11; 57-60.

19. Burns DK, Silva FG, Forde KA, Mount PM, Clark HB. Primary melanocytic schwannoma of the stomach. Evidence of dual melanocytic and schwannian differenciation in an extra-axial site in a patient without neurofibromatosis. Cancer 1983; 52; 1432-1441.

20. Erlandson RA. Melanotic schwannoma of spinal nerve origin. Ultrastruct. Pathol. 1985; 9: 123-129.

21. Miettinen M. Melanotic schwannoma coexpression of vimentin and glial fibrillary acidic protein. Ultrastruct. Pathol. 1987; 11; 39-46.

22. Di Bella C, Declich P, Assi A et al. Melanotic schwannoma of the sympathetic ganglia: a histologic, immunohistochemical and ultrastructural study. J. Neuro-oncol. 1997; 35; 149-152. 